\title{
Comparison of Sperm Functional Parameters after Cryopreservation of Human Sperm in Three Different Media
}

\author{
Sindhuja N.S. ${ }^{1}$, Manjula G. ${ }^{2}$, Sanjeeva Reddy N. ${ }^{3}$ \\ ${ }^{1}$ Lecturer in Clinical Embryology, ${ }^{2}$ Assistant Professor in Clinical Embryology, ${ }^{3}$ Professor and Head, \\ Department of Reproductive Medicine and Surgery, Sri Ramachandra Institute of Higher Education and Research, \\ Chennai-600116 \\ Emailid- ${ }^{1}$ namboori1990@gmail.com, ${ }^{2}$ manjula2000srmc@gmail.com, ${ }^{3}$ royalnsr@gmail.com \\ *Corresponding author - Sindhuja N.S.
}

Received 22 January 2019; Revised Date: 06 February 2019; Accepted 13 February 2019; Published 16 February 2019

\begin{abstract}
Semen cryopreservation is an integral part of infertility treatment in the current day scenario. Recovery of an optimal number of functionally intact spermatozoa from thawed samples has always been the main objective of semen cryopreservation technology. The aim of this study was to compare of Commercial freezing media with Glycerol egg yolk citrate and Soy lecithin media for the cryopreservation of human spermatozoa. This prospective observational study was done at the department of reproductive medicine and surgery, Sri Ramachandra University during the period of August 2015 to January 2016. Male partner of the infertile couple visiting OPD for infertility treatment with normal semen quality (Normozoospermic) were included in the study. Each semen sample was divided into three aliquots and frozen with Commercial media, Glycerol Egg Yolk Citrate (GEYC) media and soy lecithin media. Semen analysis and sperm function tests before freezing and after thawing namely Acrosome reaction, Capacitation and Mitochondrial potential was compared between the groups. There was a significant decline in motility, vitality and functional parameters of the sperms compared to fresh and frozen thawed in all the groups. However, among the frozen thawed groups, the commercial media and GEYC media were comparable in motility and viability parameters;GEYC media was superior to Commercial media in functional sperm parameter maintenance.
\end{abstract}

Keywords: semen freezing, acrosome reaction, capacitation, mitochondrial potential.

\section{Introduction}

Cryopreservation is a process where cells, whole tissues or any other substances susceptible to damage caused by chemical reactivity or time are preserved by cooling to subzero temperatures. Semen cryopreservation is a process to preserve sperm cells. The process was applied to humans during 1950 with pregnancies obtained after insemination of frozen sperm. ${ }^{[1,2]}$ Cryopreservation is known to have detrimental effects on the sperm structure and function. Recovery of an optimal number of functionally intact spermatozoa from thawed samples has always been the main objective of semen cryopreservation technology. ${ }^{[3]}$ Cryopreservation impairs sperm motility ${ }^{[4]}$ viability, ${ }^{[5]}$ penetration into cervical mucus, penetration of zona-free hamster eggs, ${ }^{[4]}$ acrosome structure, ${ }^{[6]}$ and activity of acrosome protease and acrosin. ${ }^{[7]}$ The fertilizing ability of human sperm is reduced after cryopreservation, which can be explained partially by a reduction in the percentage of normal intact acrosomes and in total acrosin activity. ${ }^{[7]}$ Efforts to improve the fertilizing ability of cryopreserved spermatozoa will be useful for clinical applications.

The principle involved in cryopreservation is the prevention of intracellular ice crystal formation and optimal dehydration of the cells. In order to prevent cellular damage due to altered solute concentration at lower temperatures, a cryoprotectant is essential. Cryoprotectants, simply by increasing the total concentration of all solutes in the system, reduce the amount of ice formed at any given temperature; but to be biologically acceptable they must be able to penetrate into the cells and have low toxicity. They also act by decreasing the freezing point of a solution by increasing the amount of salts and solutes present in the liquid phase of the sample, thereby decreasing ice formation within the spermatozoa. ${ }^{[8]}$ The survival of spermatozoa during freezing and thawing is affected by many factors. These include type and concentration of cryoprotectant, temperature at which the cryoprotectant is added, composition of the preserving medium, freezing rate and thawing temperature, and many interactions. ${ }^{[9]}$ 
Glycerol is the cryoprotectant most widely used for human semen. ${ }^{[10]}$ Lecithin has been reported to have neither a cytotoxic effect nor a negative effect on sperm motility, whereas lyophosphatidylcholine and other fatty acids have inhibitory effects on sperm motility and induce acrosomal damage. Egg yolk, a compound of lipoproteins, phospholipids, cholesterol, and various other less-abundant components, is also used in combination with Tris buffers to constitute TEST-yolk buffer (TYB) for sperm cryopreservation with a good post thaw recovery rate. ${ }^{[11,12]} \mathrm{Egg}$ yolk may play a role in reducing the deleterious effects on membrane structures of hyperosmotic salt solutions that occur during rapid cooling. ${ }^{[13]}$

Hence the aim of this study was Comparison of sperm functional parameters after cryopreservation of human sperm in three different media.

\section{Materials and methods}

This prospective observational study was done at the department of reproductive medicine and surgery, Sri Ramachandra Institute of Higher Education and Research during the period of August 2015 to January 2016. Male partner of infertile couples with normal semen quality (Normozoospermia) attending the Out Patient Department (OPD), for routine semen analysis were recruited for the study. Semen samples were analyzed manually. The mean values of the fresh semen analysis and sperm function tests are described in table 1. Men with Azoospermia, Abnormal semen parameters such as Oligozoospermia, Asthenozoospermia, Teratozoospermia and combinations of these abnormalities, highly viscous samples were excluded from the study.

\subsection{Sample collection}

Semen sample was collected by the male partner after 2-7 days of abstinence by masturbation in a sterile, non toxic wide mouthed semen collection container. The fresh semen sample was allowed to liquefy and analyzed by manual method within thirty minutes according to WHO 2010 guidelines. ${ }^{[14]}$

Table 1: Mean values of analysis of fresh sample. The tables shows the inclusion of only normal sample for the study

\begin{tabular}{|l|c|}
\hline Parameter(unit) & $\begin{array}{c}\text { Fresh Sample } \\
\text { (Mean } \pm \text { SD) }\end{array}$ \\
\hline Volume(ml) & $2.58 \pm 0.6$ \\
\hline Concentration(million/ml) & $40.58 \pm 21.9$ \\
\hline Total Motility (\%) & $57.40 \pm 9.4$ \\
\hline Progressive Motility (\%) & $35.34 \pm 8.6$ \\
\hline Non Progressive Motility (\%) & $21.86 \pm 5.2$ \\
\hline Immotile (\%) & $43.68 \pm 10.8$ \\
\hline Viability (\%) & $69.52 \pm 11.2$ \\
\hline Normal Morphology (\%) & $5.06 \pm 1.5$ \\
\hline Head defects (\%) & $50.76 \pm 6.5$ \\
\hline Midpiece defects (\%) & $43.64 \pm 6.7$ \\
\hline Tail defects (\%) & $0.30 \pm 0.5$ \\
\hline Acosome Intact (\%) & $66.60 \pm 11.1$ \\
\hline Capacitated (\%) & $19.56 \pm 8.1$ \\
\hline Mitochondrial Potential (\%) & $67.80 \pm 7.8$ \\
\hline
\end{tabular}

\subsection{Assessment of sperm concentration}

Sperm concentration was assessed by placing a drop (10 $\mu$ l) of sperm suspension on a Makler's counting chamber and the sperm concentration was expressed in millions/ml by observing under light microscope.

\subsection{Assessment of sperm motilty:}

Sperm motility was assessed manually by placing a drop $(10 \mu \mathrm{l})$ of sperm suspension on a clean glass slide above which a cover slip was carefully placed. The number of motile sperm were graded and counted separately within 30 minutes from the time of sample collection under light microscope (40X). The motility was graded into 3 categories namely: Progressive, Non Progressive. Immotile and was expressed in percentage (WHO 2010). ${ }^{[14]}$

\subsection{Sperm morphology}

Sperm morphology was assessed by making a thin smear of sperm suspension on a clean glass slide. The slides were stained by Diff Quik staining technique and observed under light microscope. Percentage of sperm with normal morphology was calculated (WHO 2010). ${ }^{[14]}$

\subsection{Sperm viability}

Sperm viability was assessed by the Hypo Osmotic Swelling Test (HOST). The sample preparation was done by mixing sperm suspension and hypo osmotic swelling solution in the ratio 1:10 and incubating at $37^{\circ} \mathrm{C}$ for 30 minutes. It was then observed at a magnification of 40X under light microscope. A total of 200 spermatozoa were counted according to the WHO criteria (2010). ${ }^{[14]}$ The percentage of swollen spermatozoa was taken as the measure for number of intact membranes and hence the sperm's viability.

\subsection{Acrosome reaction and capacitation reaction:}

Assessed by the Chlortetracyclin Assay (CTC). Sperm suspension from fresh ejaculate was washed twice using sperm wash supplemented with Human Serum Albumin (HSA). The resultant pellet was resuspended with wash media. The reaction was initiated by mixing $10 \mu 1$ of sperm suspension with $10 \mu$ CTC solution $(2 \mathrm{mM})$ in the dark. After 15 seconds the reaction was stopped by adding $10 \mu 1$ glutaraldehyde solution (12.5\%). A drop $(10 \mu 1)$ of this mixture was placed on a glass slide and covered with cover slip. The sperm fluorescence pattern was evaluated under the fluorescent microscope and percentage of acrosome intact and capacitated sperms were assessed.

\subsection{Mitochondrial potential}

Assessed by using the Rhodamine 123 stain. The semen was centrifuged and resuspended in sperm wash media containing $10 \mu \mathrm{g} / \mathrm{ml}$ of Rhodamine 123 . The sperm suspension was incubated at $37^{\circ} \mathrm{C}$ for 20 minutes and washed twice with media. A drop of the suspension was placed on a clean glass slide and observed under fluorescent microscope to assess mitochondrial integrity. The number of spermatozoa showing fluorescence was counted and expressed in percentage.

\subsection{GEYC Cryoprotectant was prepared in house:}

$1.5 \mathrm{~g}$ of glucose and $1.3 \mathrm{~g}$ of sodium citrate tribasic dehydrate to 65 $\mathrm{ml}$ of sterile purified water was added After complete dissolution, $15 \mathrm{ml}$ of glycerol and $1.3 \mathrm{~g}$ of glycine was added and mixed thoroughly. When dissolved, solution is filtered through a $0.45 \mathrm{~mm}$ pore filter. To this, $20 \mathrm{ml}$ of fresh egg yolk (pathogen free eggs). The whole suspension is kept in a water bath at $56^{\circ} \mathrm{C}$ for 40 minutes with occasional swirling. The $\mathrm{pH}$ of the solution was maintained within 6.8-7.2.

\subsection{Soy Lecithin cryoprotectant - prepared in house:}

The effect of different concentrations of soy lecithin was compared prior to the beginning of the study and a concentration of $1.5 \%$ was found to give results better than $0.5 \%, 1 \%$ and $2 \%$ of soy lecithin. 
Hence $1.5 \%$ of soybean lecithin was used in this study. The basic extender composed of $70 \mathrm{mM}$ Fructose, $85 \mathrm{mM}$ citric acid, $5 \%$ glycerol $(\mathrm{v} / \mathrm{v})$. The osmotic pressure and $\mathrm{pH}$ were set at 420 $\mathrm{mOsm} / \mathrm{kg}$ and 6.8 respectively. For final preparation of extender, $450 \mathrm{mg}$ soybean lecithin was added and shaken for ten minutes.

\subsubsection{Cryopreservation protocol}

After proper labeling, liquefied semen samples were divided into three aliquots. Each aliquot was mixed with equal volume of cryoprotectant (One aliquot with GEYC media, one aliquot with Soy Lecithin media, one aliquot with Commercial Media) in a drop wise manner with gentle mixing. The mixture was transferred to a sterile cryovial and placed in $4^{\circ} \mathrm{C}$ for 10 minutes, at $-4^{\circ} \mathrm{C}$ for 5 minutes, liquid nitrogen vapours $\left(-165^{\circ} \mathrm{C}\right)$ for $2-3$ minutes and finally plunged into the liquid nitrogen dewar $\left(-196^{\circ} \mathrm{C}\right)$.

\subsubsection{Thawing}

The cryovials were removed from the liquid nitrogen dewar 7 days after freezing and immediately placed under running tap water for 10 minutes until the samples were thawed completely. The samples were mixed with $1 \mathrm{ml}$ of buffered solution and centrifuged at 1500rpm for 5 mins to remove the cryoprotectant. The sperm pellet was washed with buffer media supplemented with HAS before using for further analysis.

Once the samples were thawed completely, they were subjected to all the tests that were performed in the fresh semen sample. The results of the analysis and sperm function tests were compared between the fresh and post thaw samples.

\section{Results}

The results of our study has shown that there was a significant difference in motility parameters between pre freeze $(57.4 \%)$ and post thaw samples while using GEYC media (40.7\%), Soy lecithin media (32.2\%) and the commercial media (41.9\%) [P value<0.005]. The frozen thawed parameters of sperms cryopreserved in commercial media and glycerol egg yolk citrate are described in table 2.

The comparison of the three media with the fresh sample is illustrated in figure 1.

Table 2: Comparison of sperm parameters between those Frozen-thawed with Commercial Freezing Media and Glycerol Egg Yolk Citrate

\begin{tabular}{|l|c|c|c|}
\hline Parameter (unit) & $\begin{array}{c}\text { Commercial } \\
\text { media-Post } \\
\text { thaw }\end{array}$ & $\begin{array}{c}\text { GEYC } \\
\text { media-Post } \\
\text { thaw }\end{array}$ & p Value \\
\hline $\begin{array}{l}\text { Total Motility } \\
(\%)\end{array}$ & $41.9 \pm 11.3$ & $40.7 \pm 12.5$ & 0.872 \\
\hline $\begin{array}{l}\text { Progressive } \\
\text { Motility (\%) }\end{array}$ & $23.6 \pm 7.7$ & $24.7 \pm 8.8$ & 0.791 \\
\hline Viability (\%) & $58.9 \pm 11.9$ & $59.8 \pm 13.8$ & 0.950 \\
\hline $\begin{array}{l}\text { Acrosome Intact } \\
(\%)\end{array}$ & $53.2 \pm 13.8$ & $59.7 \pm 12.1$ & $0.028 *$ \\
\hline Capacitated (\%) & $39.7 \pm 11.6$ & $32.1 \pm 9.8$ & $0.003 *$ \\
\hline $\begin{array}{l}\text { Mitochondrial } \\
\text { Potential (\%) }\end{array}$ & $55.7 \pm 7.5$ & $57.1 \pm 7.5$ & 0.644 \\
\hline
\end{tabular}

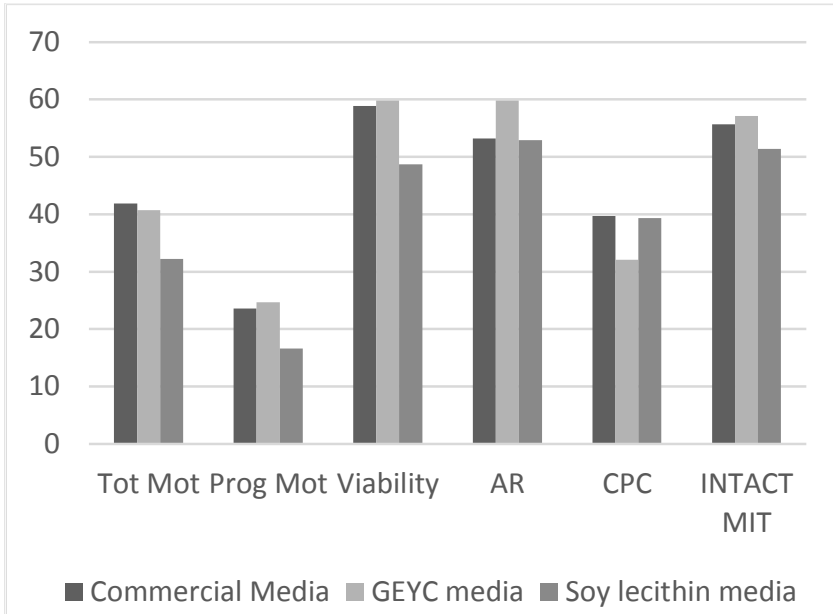

Figure1: Comparison of the three different media with the fresh sample

(Tot mot-total motility, prog mot- progressive motility, ARacrosome reaction, CPC- capacitated sperms, Intact mit- sperms with intact mitochondria)

On comparing the Commercial media with $1.5 \%$ soy lecithin media the motility [Commercial (41.9\%); Soy lecithin (32.3\%), P value $<0.005$ ] and viability [Commercial (58.9\%); Soy lecithin $(48.7 \%) \mathrm{P}$ value $<0.01]$ parameters were conserved better in Commercial media in our study. Also the mitochondrial potential [Commercial (55.7\%); Soy lecithin (51.4\%)] [P value=0.018] was found to be superior in sperms frozen thawed with the commercial media. The parameters are tabulated in table 3 .

Table 3: Comparison of sperm parameters between those Frozen-thawed with Commercial Freezing Media and 1.5\% Soy Lecithin media

\begin{tabular}{|l|c|c|c|}
\hline Parameter (unit) & $\begin{array}{c}\text { Commercial } \\
\text { media-Post } \\
\text { thaw }\end{array}$ & $\begin{array}{c}\text { 1.5\%Soy } \\
\text { Lecithin } \\
\text { post thaw }\end{array}$ & p Value \\
\hline $\begin{array}{l}\text { Total Motility } \\
(\%)\end{array}$ & $41.9 \pm 11.3$ & $32.2 \pm 13.9$ & $0.001^{*}$ \\
\hline $\begin{array}{l}\text { Progressive } \\
\text { Motility (\%) }\end{array}$ & $23.6 \pm 7.7$ & $16.6 \pm 8.8$ & $0.005^{*}$ \\
\hline Viability (\%) & $58.9 \pm 11.9$ & $48.7 \pm 16.0$ & $0.001^{*}$ \\
\hline $\begin{array}{l}\text { Acrosome Intact } \\
(\%)\end{array}$ & $53.2 \pm 13.8$ & $53.9 \pm 12.0$ & 0.947 \\
\hline Capacitated (\%) & $39.7 \pm 11.6$ & $39.3 \pm 12.4$ & 0.986 \\
\hline $\begin{array}{l}\text { Mitochondrial } \\
\text { Potential (\%) }\end{array}$ & $55.7 \pm 7.5$ & $51.4 \pm 8.4$ & $0.018^{*}$ \\
\hline
\end{tabular}

*Significant

In this study the sperms frozen with Egg Yolk Media were found to have significantly higher percentage of motile [Egg yolk (40.7\%); Soy lecithin (32.2\%)] and viable sperms [Egg yolk (59.8\%); soy lecithin $(53.9 \%)$ ] than those frozen thawed with soy lecithin media. Also, a higher proportion of uncapacitated sperms [Egg yolk (68\%); Soy lecithin (61\%)] and sperms with good mitochondrial potential [Egg yolk (57.1\%); Soy lecithin (51.4\%)] was found with Egg yolk media. The acrosome status of the sperms frozen thawed with both the media was comparable. 
Table 4: Comparison of sperm parameters between those Frozen-thawed with Commercial Freezing Media and 1.5\% Soy Lecithin media

\begin{tabular}{|l|c|c|c|}
\hline Parameter (unit) & $\begin{array}{c}\text { GEYC } \\
\text { media-Post } \\
\text { thaw }\end{array}$ & $\begin{array}{c}\mathbf{1 . 5 \% S o y} \\
\text { Lecithin post } \\
\text { thaw }\end{array}$ & p Value \\
\hline $\begin{array}{l}\text { Total Motility } \\
(\%)\end{array}$ & $40.7 \pm 12.5$ & $32.2 \pm 13.9$ & $0.003^{*}$ \\
\hline $\begin{array}{l}\text { 'Progressive } \\
\text { Motility (\%) }\end{array}$ & $24.7 \pm 8.8$ & $16.6 \pm 8.8$ & $0.005^{*}$ \\
\hline Viability (\%) & $59.8 \pm 13.9$ & $48.7 \pm 16.0$ & $0.005^{*}$ \\
\hline $\begin{array}{l}\text { Acrosome Intact } \\
\text { (\%) }\end{array}$ & $59.8 \pm 12.1$ & $53.9 \pm 12.0$ & 0.09 \\
\hline Capacitated (\%) & $32.1 \pm 9.8$ & $39.32 \pm 12.4$ & $0.004^{*}$ \\
\hline $\begin{array}{l}\text { Mitochondrial } \\
\text { Potential (\%) }\end{array}$ & $57.1 \pm 7.5$ & $51.4 \pm 8.4$ & $0.002^{*}$ \\
\hline
\end{tabular}

*Significant

\section{Discussion}

As we have included only normozoospermic samples in our study, most of the patients (64\%) were in the age group of 30-35years. This correlates with a study by Stone et al who analyzed sperm samples from 5081 men aged between 16 and 72 years, and found that the sperm quality was predominantly normal in 30-35 years compared to those in age groups above 35 years. 15 Similar to the worldwide statistics, in this study $76 \%$ of the patients had primary infertility. As male factor infertility was excluded, all our patients belonged to the female factor or unexplained factor groups. In India, approximately $50 \%$ of cases are due to female factor, and $20-30 \%$ of cases are due to male factor and the remaining $20-30 \%$ is due to a combination of male and female factors and unexplained infertility. ${ }^{[16]}$

Among the three buffers, samples frozen with GEYC media showed higher motility. This result was in accordance with a study by Hallak et al who found that Glycerol with Egg yolk rather than Glycerol alone gave better post thaw results. In their study, they have compared the action of two buffers: Egg yolk buffer and glycerol as cryoprotectants. Their study showed that there was a significant decrease in percentage of motile sperms between pre freeze [44\%] and post thaw [Egg yolk (10.5\%); Soy lecithin (5\%)] in both the buffers. However the Egg yolk buffer gave better results than Glycerol. ${ }^{[17]}$

Mohsen Sharafi et al have found that there was no significant difference on comparing the motility [Commercial (50.1\%); Soy lecithin (52.6\%)], viability [Commercial (47.4\%); Soy Lecithin $(49.1 \%)$ ], capacitation status [Commercial $(59.8 \%)$, Soy lecithin $(61.1 \%)$ ] and acrosome status [Commercial $(31.8 \%)$, Soy lecithin $(27.8 \%)]$ between commercial media and Soy lecithin media. ${ }^{[18]}$

Although our study findings describe that GEYC media is superior to soy lecithin media; In a similar study, Michael et al compared the post thaw parameters of human sperm cryopreserved with Egg Yolk media and Soy lecithin media. They found that Soy lecithin could replace egg yolk for cryopreservation of human sperm without adversely affecting post thaw motility [Soy lecithin (28.8\%); Egg yolk (36.4\%)], morphology [Soy lecithin (3.8\%); Egg yolk (3.9\%)], sperm DNA integrity [Soy lecithin (21.3\%); Egg yolk $(21.5 \%)$ ], or sperm binding to hyaluronate [Soy Lecithin $(85.4 \%)$; Egg yolk (85.2\%)]. ${ }^{[19]}$

\section{Conclusion}

Among GEYC and soy lecithin media, sperms frozen thawed with GEYC media showed better quality and functional capacity. The motility, viability and functional capacity of sperms frozen thawed with GEYC media and commercial media were similar. Hence due to cost effectiveness and in house reproducibility, GEYC could be an effective replacement for commercial media.

\section{References}

[1] Sanger WG, Olson JH, Sherman JK: Semen cryobanking for men with cancer. Fertility and sterility 1992; 58(5): $1024-1027$

[2] AngerJT, GilbertBR, GoldsteinM: Cryopreservation of sperm: indications, methods and results. J Urol. 2003 Oct;170(4):1079-84.

[3] Bunge RG, Keettel WC, Sherman JK: Clinical use of frozen semen. Fertil Steril 1954;5:520-529.

[4] Crister JK, Arneson BWA, Aaker DV. (1987) Cryopreservation of human spermatozoa: Post thaw choronology of motility and of zona-free hamster ova penetration. Fertil Steril 1987; 47:980-4.

[5] Alwarez JG, Storey BT. Evidence that membrane stress contributes more than lipid peroxidation to sublethal cryodamage in cryopreserved human sperm: glycerol and other polyols as sole cryoprotectants, J.Androl.14; 199209.

[6] Mc Laughlin EA, Ford WC, Hull MG. Effects of cryopreservation on the human sperm acrosome and its response to A3187: Reprod Fertil 1993;99(1):71-6.

[7] Mack, S.R. and Zaneveld, L.J. Acrosomal enzymes and ultrastructure of unfrozen and cryotreated human spermatozoa. Gamete Res 1987; 18: 375-383.

[8] Royere, D., Barthelemy, C., Hamamah, S. and Lansac, J. Cryopreservation of spermatozoa: a 1996 review. Hum. Reprod. Update 1996; 2: 553-559.

[9] P. Stanic, M. Tandara, Z. Sonicki, V. Simunic, B. Radakovic, and E. Suchanek, Comparison of protective media and freezing techniques for cryopreservation of human semen, European Journal of Obstetrics Gynecology and Reproductive Biology 2000;91:1: 6570.

[10] Sherman jk. Dimethyl sulfoxide as a protective agent during freezing and thawing of human spermatozoa; Proc Soc Exp Biol Med. 1964 Oct;117:261-4

[11] Prins GS, Weidel L. A comparative study of buffer systems as cryoprotectants for human spermatozoa. Fertil Steril. 1986 Jul; 46(1):147-9.

[12] Weidel L, Prins GS.Cryosurvival of human spermatozoa frozen in eight different buffer systems. J Androl. 1987 Jan-Feb;8(1):41-7.

[13] Holt WV1, Head MF, North RD. Freeze-induced membrane damage in ram spermatozoa is manifested after thawing: observations with experimental cryomicroscopy. Biol Reprod. 1992 Jun; 46(6):1086-94.

[14] WHO Manual of Semen Analysis 2010 edition.

[15] Stone BA, Alex A, Werlin LB, Marrs RP. Age thresholds for changes in semen parameters in men. Fertil Steril. 2013;100:952-8.

[16] AshokAgarwal, Aditi Mulgund, Alaa Hamada, Michelle Renee Chyatte. A unique view on male infertility around the globe. Reprod Biol Endocrinol. 2015; 13: 37. 
[17] Jorge Hallak, Ashok Agarwal, Rakesh K Sharma. Cryopreservation of human sperm-comparison of test yolk buffer and Glycerol. Int J Fertil.2000;45:38-42

[18] Mohsen Sharafi, Mohsen Forouzanfar. In Vitro Comparison of Soybean Lecithin Based-Extender with Commercially Available Extender for Ram Semen Cryopreservation. International Journal of Fertility and Sterility. 2009 Nov; 33: 149-152.

[19] Reed ML,Ezeh PC, Hamic A, Thompson DJ, Caperton CL. Soy lecithin replaces egg yolk for cryopreservation of human sperm without adversely affecting post thaw motility, morphology, sperm DNA integrity, or sperm binding to hyaluronate. Fertil Steril. 2009 Nov; 92(5):1787-90
Author Profile

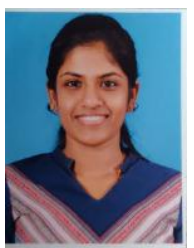

Dr. Sindhuja has completed her M.B.B.S and has pursued masters in clinical embryology from Sri Ramachandra Institute of Higher Education and Research. She is currently a faculty in clinical embryology in the Department of Reproductive Medicine and Surgery at Sri Ramachandra Institute of Higher Education and Research. Her areas of interest include all aspects of andrology and embryology. 